\title{
Integrating science and practice on placements
}

Authors: Jason Hall, Paul Buckley*, Joanne Hayes*, Ellen Schafheutle, Ruth Ledder, Gavin Humphries, Victoria Silkstone. Manchester Pharmacy School, The University of Manchester and *Stockport NHS Foundation Trust

Background: Providing an entire cohort of students with the opportunity to learn about manufacturing in a real life setting has always been a challenge for pharmacy schools. Helping them to apply this learning to patient care is equalling challenging. This abstract describes an initiative where students visit manufacturing departments in a hospital and meet with patients to discuss how the medicines are used. This initiative aims to support integration by demonstrating the application of science to the preparation and testing of a range of formulations and appreciating the formulations form the patient's perspective.

Description of work: Academic staff worked closely with colleagues from hospital pharmacy to produce a workbook to structure the student visit to the aseptic unit, manufacturing unit, quality control laboratories and pharmacy led outpatient clinics. Students attended the hospital in groups of eight and visited each unit/clinic in pairs. The activities in the workbook tied in closely with existing teaching sessions delivered in the University on microbiology, chemistry and law [manufacturing, independent prescribing] so the students could see how the science applies to practice to strengthen and underpin learning. The workbook was not assessed although material from the visit and the work book was examinable.

Proposed evaluation: Students had the opportunity to provide feedback at the end of the visit via an anonymous questionnaire and also back at the university using Turnitin $\circledast$ Response devices. Early analysis of this feedback has been very positive.

Conclusion: This visit allowed students to see how medicines are prepared, tested and used in real life situations. Its strengths lie not just in the exposure to experts working in the field of classical manufacture, aseptic manufacture, quality control and in the clinic but also in the integration of science and practice and integration of academic study with patient care and pharmacy practice. 